\title{
Involuntary transfer of a top-down attentional set into the focus of attention: Evidence from a contingent attentional capture paradigm
}

\author{
Katherine Sledge Moore and Daniel H. Weissman \\ University of Michigan, Ann Arbor, Michigan
}

\begin{abstract}
In the present study, we investigated whether involuntarily directing attention to a target-colored distractor causes the corresponding attentional set to enter a limited-capacity focus of attention, thereby facilitating the identification of a subsequent target whose color matches the same attentional set. As predicted, in Experiment 1 , contingent attentional capture effects from a target-colored distractor were only one half to one third as large when subsequent target identification relied on the same (vs. a different) attentional set. In Experiment 2, this effect was eliminated when all of the target colors matched the same attentional set, arguing against bottomup perceptual priming of the distractor's color as an alternative account of our findings. In Experiment 3, this effect was reversed when a target-colored distractor appeared after the target, ruling out a feature-based interference account of our findings. We conclude that capacity limitations in working memory strongly influence contingent attentional capture when multiple attentional sets guide selection.
\end{abstract}

In order to protect limited-capacity information processing systems, attention is posited to direct processing resources toward stimuli that are important for achieving current behavioral goals (Desimone \& Duncan, 1995; Posner \& Rothbart, 2007). Such goal-directed attention is thought to be made possible by representations of behavioral goals that specify the attentional settings that are needed to optimize the processing of behaviorally relevant stimuli (Folk, Remington, \& Johnston, 1992). For example, the goal of searching for a lemon at the grocery store is usually linked to searching for the color yellow. Once such attentional settings are specified, top-down signals are posited to bias sensory processing in favor of stimuli whose features match these settings (Corbetta \& Shulman, 2002), thereby enhancing the ability of relevant stimuli to gain access to limited-capacity systems.

Although selective attention usually helps to prevent irrelevant stimuli from gaining access to limited-capacity systems, sometimes it has the opposite effect. For example, when one is searching for a lemon at the grocery store, all of the yellow items in the fruits and vegetables sectionincluding lemons, peppers, and squash - may attract attention. Put another way, attention is often involuntarily directed to an irrelevant stimulus that possesses a feature (e.g., a particular color) that matches a top-down attentional set. In the laboratory, contingent attentional capture refers to a phenomenon in which involuntarily directing attention to an irrelevant stimulus that possesses a taskrelevant feature impairs the identification of a subsequent target stimulus (Folk et al., 1992). Contingent capture ef- fects have been observed in spatial cuing (Bacon \& Egeth, 1994; Folk et al., 1992; Gibson \& Kelsey, 1998), visual search (Olivers, 2008), and rapid serial visual presentation (RSVP) paradigms (Folk, Leber, \& Egeth, 2002, 2008; Leblanc, Prime, \& Jolicœur, 2008; Serences et al., 2005), and are thought to arise because a distractor whose properties match an attentional set is processed more deeply, as though it were a target (Folk et al., 2002). Because this deeper processing is serial and capacity limited, it impairs the identification of a target that appears later in time or at a different location. Thus, although selective attention usually limits the processing of irrelevant stimuli, it sometimes enhances such processing.

Although the phenomenon of contingent attentional capture is firmly established in the literature, relatively little is known about such capture in the context of maintaining multiple attentional sets. Current models of working memory posit that up to four representations can be maintained simultaneously (Cowan, 2001; Jonides et al., 2008; Oberauer, 2002). Consistent with such models, participants can maintain rules for at least three tasks in task switching experiments (Mayr \& Keele, 2000; Monsell, 2003). Moreover, in visual attention tasks, contingent capture effects can be produced by irrelevant stimuli that match either of two concurrently maintained attentional sets (Adamo, Pun, Pratt, \& Ferber, 2008; Ansorge \& Heumann, 2003). Nonetheless, it remains unclear whether some contingent attentional capture effects are different when participants maintain multiple attentional sets than when they maintain just a single attentional set. 
Evidence to suggest that contingent attentional capture effects may indeed differ in these two situations comes from numerous findings indicating that it is possible to focus attention on only one representation in working memory at a time (Oberauer, 2002, 2003). First, when multiple items are maintained in working memory, the time it takes to identify the most recently presented or rehearsed item is much shorter than the time needed to identify other items, suggesting that this item is alone in the focus of attention (McElree, 2001). Second, in experiments that investigate the updating of multiple counters, participants are faster when updating the same counter sequentially than when switching between counters, indicating that only one counter can be focused on at a time (Berti, 2008; Garavan, 1998). Third, task switching studies show that participants are faster and more accurate when repeating the same task than when switching from one task to another, suggesting that participants can focus only on one task set at a time (Hsieh \& Allport, 1994; Monsell, 2003). And fourth, participants experience a smaller "attentional blink" (i.e., decreased accuracy when identifying the second of two rapidly presented targets) when the same attentional set is used to identify the two targets than when different attentional sets are used, regardless of whether the attentional sets are defined by conceptual category (e.g., letters vs. digits) (Juola, Botella, \& Palacios, 2004) or by location (Vachon, Tremblay, \& Jones, 2007). These findings suggest that voluntarily directing attention to a particular stimulus or task causes the corresponding attentional set to enter the limited-capacity focus of attention, thereby enhancing future behaviors that rely on the same set.

In the present study, we investigated whether involuntarily directing attention to a distractor that possesses a target-defining color also causes the corresponding attentional set to enter the focus of attention. According to this enhancement hypothesis, an integral component of deeply processing a target-colored distractor is moving that item's attentional set into the focus of attention until the item has been successfully identified. In line with this possibility, various theories of visual search posit that detecting a potential target leads to the recruitment of working memory processes that attend, identify, or otherwise consolidate that item (see Dux \& Marois, 2009). And, consistent with this view, it has been shown that information about distractors is transferred into working memory during the performance of capture tasks (Belopolsky, Kramer, \& Godijn, 2008). However, none of the existing contingent attentional capture studies requiring participants to maintain multiple attentional sets has explored whether one set ever becomes prioritized, or enhanced, over the others during the time course of a single trial (Adamo et al., 2008; Ansorge \& Heumann, 2003).

The enhancement hypothesis predicts that contingent attentional capture effects that are caused by deeply processing a target-colored distractor should be smaller when a target appearing soon afterward matches the same (vs. a different) attentional set. Deeply processing a targetcolored distractor should always impair the identification of a subsequent target. However, this impairment should be reduced when target identification relies on the same attentional set and therefore does not involve loading a different attentional set into the focus of attention. This view is consistent with the findings from working memory, task switching, and attentional blink studies that we reviewed earlier, all of which demonstrate a relative enhancement in performance when a target can be identified using the attentional set that is currently inside the focus of attention. As an everyday example, when one is searching for both lemons and garlic at the grocery store, deeply processing a yellow pepper may cause the "lemon" attentional set to temporarily enter the focus of attention. While the "lemon" attentional set is prioritized, it could become comparatively easier to notice a nearby lemon than a garlic bulb, even though it may still be harder to notice either item than if the yellow pepper had not captured attention.

The enhancement hypothesis may appear to contradict previous findings from visual search tasks indicating that the manner in which attentional sets are used in one trial can influence how they are used in the next trial (Belopolsky, Schreij, \& Theeuwes, 2010). Specifically, although intertrial priming of attentional sets is not always observed (Ansorge \& Horstmann, 2007; Ansorge, Horstmann, \& Carbone, 2005), in some studies, identifying a distractor that possesses a target-defining feature (e.g., a particular color) in one trial is associated with a decreased attentional bias toward that feature in the next trial (Lleras, Kawahara, \& Levinthal, 2009; Olivers \& Humphreys, 2003). The present experiments differed from these prior studies, however, because they investigated within-trial changes to attentional control settings. Thus, as we will argue in greater detail in the General Discussion section, the enhancement hypothesis does not contradict prior findings regarding intertrial priming effects in visual search tasks.

It is also important for us to note that the enhancement hypothesis makes no explicit assumptions about the manner in which multiple attentional sets are maintained during active search for a target stimulus (i.e., before an item possessing a target-defining feature is detected). In some models, attentional sets are maintained in working memory (see, e.g., Olivers \& Meeter, 2008). However, there is also evidence that attentional sets can influence visual search even when they are not actively maintained in working memory (e.g., Thompson, Underwood, \& Crundall, 2007). In either case, the enhancement hypothesis posits that the detection of a potential target (including a distractor that possesses a target-defining feature) leads the attentional set corresponding to that item to enter the limited-capacity focus of attention.

Finally, although the enhancement hypothesis posits an interaction between attention and working memory, it does not speak directly to an active debate concerning this interaction. Specifically, there is an ongoing controversy over whether people automatically orient attention to stimuli whose features match the contents of working memory, but that are not the targets of an ongoing search task (e.g., Houtkamp \& Roelfsema, 2006; Olivers, 2009; Soto, Heinke, Humphreys, \& Blanco, 2005; Woodman \& Luck, 2007). In contrast, the enhancement hypothesis assumes that people automatically orient attention to stimuli 
whose features match the contents of working memory (i.e., an attentional set within the focus of attention), and that are the targets of an ongoing search task. As others have noted (e.g., Olivers, 2009), this assumption is not related to the current debate, and data to support this assumption have been reported in numerous prior studies of contingent attentional capture (e.g., Folk et al., 2002; Folk et al., 1992; Leblanc et al., 2008; Serences et al., 2005).

\section{EXPERIMENT 1}

In Experiment 1, we tested the enhancement hypothesis by instructing participants to search a central RSVP stream for letters that were presented in either of two possible target colors (e.g., orange and green). The presence of irrelevant colors in the central RSVP stream made it necessary for participants to maintain two attentional sets, one for each target color. Some targets in the central RSVP stream were preceded by a colored distractor that appeared in either of two peripheral RSVP streams. As in prior studies, we defined contingent attentional capture as lower target identification accuracy when a target-colored (e.g., orange) distractor preceded a target than when a non-target-colored (NTC; e.g., lavender) distractor preceded a target (Folk et al., 2002, 2008; Serences et al., 2005; Visser, Bischof, \& Di Lollo, 2004). Consistent with the enhancement hypothesis, we predicted that contingent attentional capture effects would be smaller when a targetcolored distractor's color (e.g., green) matched the same attentional set as the upcoming target (e.g., green) than when it matched a different attentional set (e.g., orange).

\section{Method}

Participants. Twenty-eight University of Michigan students (15 female) participated in exchange for either course credit or $\$ 10$. All of the participants (age range: 18-30 years) reported normal or corrected vision and had no history of neurological injury or disease. Each gave informed written consent in accordance with the University of Michigan Behavioral Sciences Institutional Review Board requirements.

Procedure and Design. Our task was similar to those in other contingent attentional capture experiments that have made use of RSVP displays (Folk et al., 2002, 2008; Serences et al., 2005). Participants viewed three RSVP streams. In the central stream, letters appeared in five or six different colors (see the Apparatus and Stimuli section). In two peripheral RSVP streams, most of the letters were gray. Depending on the block, participants searched for letters in the central RSVP stream that appeared in one target color (set size 1) or in either of two possible target colors (set size 2). Participants indicated whether target-colored letters in the central RSVP stream were from the first or the second half of the alphabet, respectively, by pressing either the " $\mathrm{J}$ " or the "K" key on a computer keyboard with the index or middle finger of their right hand. The first keypress logged within 2,200 msec following a target was recorded as the response to that target. The time between targetcolored letters in the central RSVP stream varied randomly from 2,320 to $4,060 \mathrm{msec}$. Every 32 trials, participants were given a selfpaced break. Figure 1A provides a schematic of the task.

Both set size blocks contained the following trial types: (1) target alone, in which a target was not preceded by a distractor, (2) sametarget-colored (STC) distractor, in which a target was preceded by a distractor of the same color, and (3) NTC distractor, in which a target was preceded by an NTC distractor. Set size 2 had an additional trial type: different-target-colored (DTC) distractor, in which a target was preceded by a target-colored distractor whose color differed from that of the target. In STC, NTC, and DTC trials, the distractor appeared one to four frames before the target, which corresponded, respectively, to stimulus onset asynchronies (SOAs) of 116, 233, 350 , and $466 \mathrm{msec}$.

Of importance, our design ensured that any performance differences between STC and DTC trials would reflect varying amounts of contingent attentional capture rather than varying amounts of response congruency in these trial types. Specifically, in all trial types with distractors (i.e., STC, NTC, and DTC), whether the targetcolored distractor and the upcoming target were from the same or different halves of the alphabet was orthogonal to whether the targetcolored distractor and the target had the same or different colors. Thus, potential effects of response congruency in the present design were not confounded with our measures of attentional capture.

In addition to the critical trial types above, occasional catch trials (in which the display paused for 1,000 msec on an item) measured whether participants were successfully maintaining the target color(s) in each set size block. In target-catch trials, the display paused on a target-colored letter that participants were supposed to identify. In non-target-catch trials, the display paused on an NTC letter that participants were not supposed to identify. The main purpose of the catch trials was to ensure that participants were equally capable of maintaining one and two target colors. With the display paused for $1,000 \mathrm{msec}$, we eliminated the encoding limitations that were imposed by the rapid speed and immediate masking of targets in the RSVP stream, thereby providing a relatively pure test of color memory. We reasoned that if participants maintained one and two target colors equally well, then a difference in the amount of contingent capture measured in set size 1 and set size 2 could not be attributed to an underlying difference in the ability to maintain one and two attentional sets.

There were 288 trials in set size 1: 48 target alone, 48 catch (24 target, 24 nontarget), 96 NTC (4 SOAs, 24 per SOA), and 96 STC (4 SOAs, 24 per SOA). There were also 288 trials in set size 2: 48 target alone, 48 catch, 64 NTC (4 SOAs, 16 per SOA), 64 STC (4 SOAs, 16 per SOA), and 64 DTC (4 SOAs, 16 per SOA).

Each set size block began with instructions explaining the task, followed by 48 practice trials. During the practice trials, the display began slowly ( $250 \mathrm{msec}$ per item), but gradually accelerated to full speed (116 msec per item) by the 24th trial. The order in which the different set size blocks were performed was counterbalanced across participants.

Apparatus and Stimuli. Stimuli were displayed on a 19-in. Viewsonic CRT monitor with a $60-\mathrm{Hz}$ refresh rate, controlled by a Dell PC running Windows XP. The experiments were programmed using Presentation software (Neurobehavioral Systems, Inc.). A viewing distance of $80 \mathrm{~cm}$ was enforced by a chinrest. Three RSVP streams containing letters $\left(2.07^{\circ} \times 1.88^{\circ}\right)$ were presented simultaneously on a black background: One stream appeared at fixation, while two others appeared $4.22^{\circ}$ to the left and $4.22^{\circ}$ to the right of fixation. Letters appeared successively in each RSVP stream for $100 \mathrm{msec}$, followed by a blank gap lasting $16 \mathrm{msec}$. Target-colored letters and colored peripheral distractors were drawn from the beginning ( $\mathrm{A}, \mathrm{B}, \mathrm{C}, \mathrm{D}$, and $\mathrm{G})$ and the end $(\mathrm{T}, \mathrm{V}, \mathrm{X}, \mathrm{Y}$, and $\mathrm{Z})$ of the alphabet to minimize demands on decision-making processes and, consequently, to provide a sensitive measure of contingent attentional capture. Other letters in the display were drawn from the entire alphabet except I, O, and $\mathrm{W}$.

Because contingent attentional capture can be influenced by bottom-up salience (Yantis \& Egeth, 1997) and by the relative discriminability of target and distractor stimuli (Anderson \& Folk, 2010), we attempted to match our colors across several important dimensions. First, we attempted to equate the perceptual salience (i.e., luminance and saturation) of all colors in each set size block. Second, within each set size block, we attempted to make the target colors equally discriminable from each other and from the color of the NTC distractor. To accomplish these objectives, we created two six-color wheels in CIELAB color space (see Figure 2), one dark 
A Set Size 2 (Experiments 1, 2, and 3)

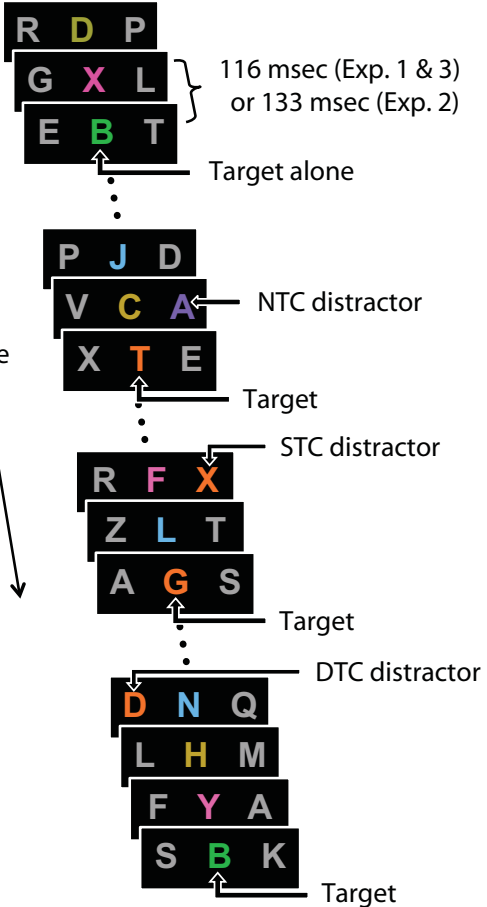

B Any-Color (Experiment 2)

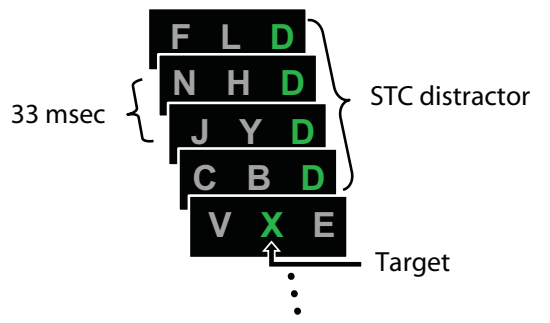

Time

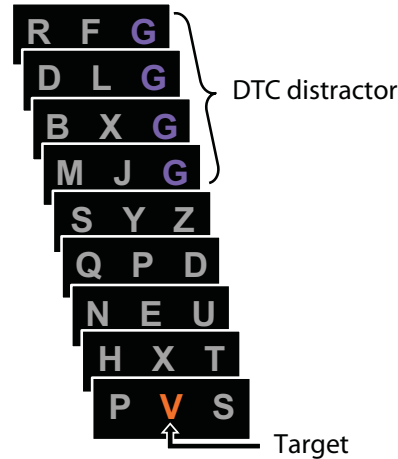

Figure 1. The contingent attentional capture tasks that we used in the present study. (A) Participants searched for target-colored letters that appeared within a heterogeneously colored, central rapid serial visual presentation (RSVP) stream while ignoring occasional colored distractors that could appear in either of two peripheral RSVP streams. Performance was measured in three types of task blocks. In one (set size 2, Experiments 1-3), participants searched for two target colors (e.g., orange and green) in the central RSVP stream. In Experiments 1 and 2, targets in the central RSVP stream appeared either alone or 1-4 items after a colored peripheral distractor (see the text for details). In Experiment 3, targets appeared alone, 1-2 items after a colored peripheral distractor, or 1-2 items before a colored peripheral distractor (not shown). Colored peripheral distractors were non-target-colored (NTC) (e.g., a lavender "V"), same-target-colored (STC), in which case the peripheral distractor's color (e.g., an orange "X") matched the same attentional set as the subsequent target's color (e.g., an orange "G"), or different-target-colored (DTC), in which case the peripheral distractor was target colored (e.g., an orange "D") but matched a different attentional set than the subsequent target's color (e.g., a green "B"). In the second type of task block (set size 1, Experiment 1, not shown), participants searched for only one target color in the central RSVP stream. Set size 1 included the same trial types as set size 2, with the exception of the DTC trial type. In both set sizes, nontarget letters in the central stream were also colored to ensure that participants were required to search for letters in the central RSVP stream that possessed the specific target color(s). Nondistractor items in the peripheral streams were gray. (B) In the third type of task block (anycolor, Experiment 2), participants identified any colored letter in the central RSVP stream. Nontarget letters in the central RSVP stream were gray, as were nondistractor letters in the peripheral RSVP streams.

$\left(\mathrm{L}^{*}=\right.$ approximately 40$)$ and one bright $\left(\mathrm{L}^{*}=\right.$ approximately 70$)$. The colors in each wheel were chosen to be as psychologically different (i.e., discriminable) from each other as possible.

A control experiment verified that our selection of colors was appropriate for testing our hypotheses about contingent attentional capture. In each trial of the control experiment, participants viewed a central RSVP stream containing 10 letters, which was presented at the same speed as in the main experiment (116 msec per item). All of the letters in the RSVP stream had the same lightness value $\left(\mathrm{L}^{*}\right)$ in CIELAB color space. Although most of the letters in the RSVP stream were gray, the second and ninth letters were assigned either the same color or two different colors from one of the color wheels. Participants judged as quickly as possible whether the colored letters were the same or different. Because participants take longer to judge two stimuli as being "different" when those stimuli are somewhat similar than when they are very different (Farell, 1985; Posner \&
Mitchell, 1967), we were able to determine how psychologically different each color was from other colors in the same wheel.

Critically, we found three nonadjacent colors in each wheel to be equally discriminable. That is, mean reaction times (RTs) to respond "different" to any two of these three colors did not significantly differ (all $p$ s $>.4, n=35$ ). These three colors from each wheel served as the target color(s) and as the NTC distractor color in one set size block. The precise mapping of each of these three colors to the target and NTC distractor stimuli was counterbalanced across participants. The remaining three colors in each wheel were randomly assigned to nontarget items that appeared in the heterogeneously colored, central RSVP stream within the same set size block. A different color wheel was used in each set size block in order to avoid proactive interference or practice effects from prior attentional sets (Leber \& Egeth, 2006; Thompson et al., 2007). The color wheel assigned to each set size block was counterbalanced across participants. 


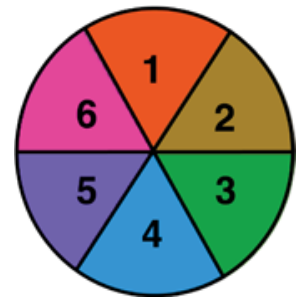

Light Color Wheel

\begin{tabular}{|c|c|c|c|c|}
\hline \multirow[b]{2}{*}{ Color } & \multicolumn{2}{|c|}{ Lignt coior whect } & \multicolumn{2}{|c|}{ Dark color willeet } \\
\hline & Name & RGB Values & Name & RGB Values \\
\hline 1 & Orange & $239,90,0$ & Brown & $122,55,0$ \\
\hline 2 & Tan & $169,132,0$ & Olive & $96,90,0$ \\
\hline 3 & Green & $48,166,0$ & Teal & $0,100,104$ \\
\hline 4 & Turquoise & $0,159,247$ & Blue & $55,29,216$ \\
\hline 5 & Lavender & $135,98,255$ & Purple & $140,0,175$ \\
\hline 6 & Magenta & $250,59,184$ & Red & $147,0,67$ \\
\hline
\end{tabular}

Figure 2. We used two color wheels to generate colored letters. All six colors in each wheel had the same lightness $\left(L^{*}\right)$ value in CIELAB space. In set size 1 and set size 2 , three equally spaced colors from a single wheel (numbers 2,4 , and 6) served as irrelevant colors in the central rapid serial visual presentation (RSVP) stream. Among the remaining three colors (1, 3, and 5), either one (set size 1) or two (set size 2) served as the target color(s), whereas the third served as the nontarget color for colored distractors in the peripheral RSVP streams. A control experiment established that, for each color wheel, all possible pairings of the target and nontarget colors (numbers 1,3 , and 5 ) were equally discriminable. Nonetheless, we counterbalanced which specific colors (numbers 1, 3, and 5) served as target and nontarget colors across participants. All gray letters in the display had the same lightness value in CIELAB space $\left(\mathrm{L}^{*}\right)$ as did colored letters appearing in the same task block. In Experiments 1 and 2, the specific color wheel that was assigned to a particular task block (set size 1 or set size 2 in Experiment 1, set size 2 or any-color in Experiment 2) was counterbalanced across participants. In the any-color condition of Experiment 2, only three equally spaced colors (numbers 1,3 , and 5) from a single color wheel appeared in the central and peripheral RSVP streams. In Experiment 3, only the light color wheel was used because performance was measured in just a single task block (set size 2).

\section{Results}

The mean accuracy was the dependent measure in all of the analyses. At the outset, we excluded 4 participants whose performance indicated that they were unable to consistently identify target-colored letters in the central RSVP stream. Specifically, each of these participants failed to respond correctly in at least $90 \%$ of target-catch trials and/or produced false alarms in more than $10 \%$ of non-target-catch trials in one or both set size blocks. Among the remaining 24 participants, catch trial accuracy was equivalent across set size [set size 1 hits vs. set size 2 hits, $96.7 \%$ vs. $96.4 \%, t(23)=0.419, p=.679$; set size 1 false alarms vs. set size 2 false alarms, $5.1 \%$ vs. $4.1 \%$, $t(23)=0.707, p=.487]$. Furthermore, performance in target-alone trials did not significantly differ in set size 1 and in set size $2[86.2 \%$ vs. $82.4 \%, t(23)=1.68, p=.11]$. Thus, it would appear that participants maintained one and two attentional sets equally well.

To test the enhancement hypothesis, we performed a repeated measures ANOVA with distractor type (STC, DTC) and SOA (116, 233, 350, $466 \mathrm{msec})$ as factors using the data from set size 2. First, in line with prior research (Folk et al., 2002, 2008), there was a main effect of SOA $[F(3,69)=31.08, p<.0001]$, indicating that participants performed more poorly at shorter than at longer SOAs. Second, consistent with the enhancement hypothesis, there was a main effect of distractor type $[F(1,23)=$ 43.56, $p<.0001]$, indicating that performance was better in STC than in DTC trials. Third, and also consistent with the enhancement hypothesis, there was an interaction between distractor type and $\operatorname{SOA}[F(3,69)=7.93, p<$ $.0001]$, because performance recovered more quickly in STC than in DTC trials (Figure 3, left). In sum, the data from set size 2 were highly consistent with the enhancement hypothesis.

We also determined whether the magnitude of contingent attentional capture as it is traditionally defined (i.e., worse performance in STC than in NTC trials) interacted with set size. To do so, we performed a repeated measures ANOVA with the factors set size $(1,2)$, distractor type (STC, NTC), and SOA (116, 233, 350, $466 \mathrm{msec})$. First, replicating prior studies of contingent attentional capture (Folk et al., 2002, 2008), we observed main effects of distractor type $[F(1,23)=36.73, p<.0001]$ and SOA $[F(3,69)=23.64, p<.0001]$, as well as an interaction between distractor type and SOA $[F(3,69)=9.47, p<$ $.0001]$. In short, performance was worse in STC than in NTC trials, and this performance decrement was larger at shorter than at longer SOAs (Figure 3). Second, a main effect of set size $[F(1,23)=8.60, p<.007]$ indicated better performance in set size 1 than in set size 2 . Third, and critically, although contingent attentional capture was present within each set size block [set size $1, F(3,69)=$ $11.08, p<.0001$; set size $2, F(3,69)=11.15, p<.0001]$, its magnitude did not vary with set size. Neither the twoway interaction between set size and distractor type nor the three-way interaction among set size, distractor type, and SOA was close to achieving significance $(F<1$ in both cases). Thus, we found no evidence to suggest that the magnitude of contingent attentional capture differed in set size 1 and set size 2 .

Finally, whether the peripheral distractor and subsequent target were from the same or different halves of the alphabet did not affect the results. Specifically, when response congruency was included as a within-participants factor in our analyses, we observed no main effects or interactions involving this factor (all $p \mathrm{~s}>.5$ ).

\section{Discussion}

Experiment 1 yielded two principal results. First, in line with the enhancement hypothesis, contingent attentional capture effects in set size 2 were significantly smaller in STC than in DTC trials. That is, when participants searched for two target colors, target identification accuracy was higher when a target was preceded by a peripheral distractor that possessed the same (vs. a different) target color. Second, the magnitude of contingent attentional capture did not vary with the number of attentional sets that participants maintained. This result is consistent with our analyses of catch trial performance, which sug- 


\section{Target alone $\longrightarrow$ NTC $\quad \square-$ STC $\quad \cdots$ N $\cdots$ DTC}

Set Size 2

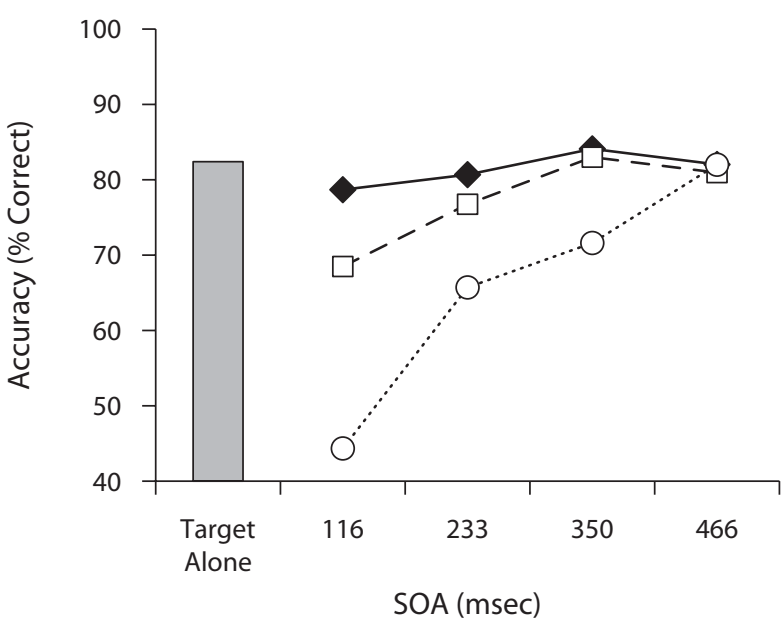

Set Size 1

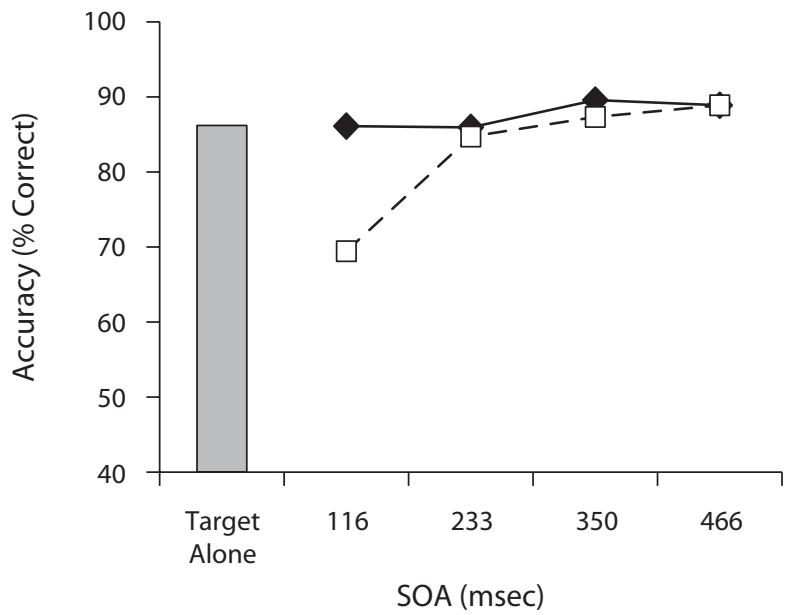

Figure 3. Data from Experiment 1. The mean target identification accuracy is plotted as a function of stimulus onset asynchrony (SOA) and distractor type in set size 2 and set size 1. In each set size, target-identification accuracy at short SOAs was lower when a target was preceded by a same-target-colored (STC) versus a non-target-colored (NTC) distractor, indicating the presence of contingent attentional capture. The magnitude of this effect did not differ in set size 1 and set size 2 . However, consistent with the enhancement hypothesis, target identification accuracy in set size 2 was lower when a target was preceded by a different-target-colored (DTC) distractor than when it was preceded by an STC distractor.

gested that participants were able to maintain one and two attentional sets equally well.

We will now consider two possible accounts of the enhancement effect (i.e., better performance in STC than in DTC trials). First, in line with the enhancement hypothesis, deeply processing a peripheral target-colored distractor may cause the corresponding attentional set (e.g., identify orange letters) to enter the focus of attention, thereby facilitating the identification of a subsequent target whose color matches the same attentional set (e.g., another orange letter) as compared with a different attentional set (e.g., a green letter). Second, and more trivial, a target-colored peripheral distractor may prime a subsequent target's color in a bottom-up fashion, so that the visual system is more prepared to see a target in that color than in a different color (Henson, 2003). We could not distinguish between these accounts in Experiment 1 because the relationship between a peripheral distractor's color and a subsequent target's color (same or different) was confounded with whether these colors matched the same or different attentional sets. Thus, we conducted a second experiment.

\section{EXPERIMENT 2}

In Experiment 2, we sought to distinguish between the enhancement and priming accounts of our findings in Experiment 1 by measuring performance in two task blocks. In one block (set size 2 from Experiment 1), participants searched for letters that appeared in either of two colors within the central RSVP stream. Because distractor items in the central RSVP stream were heterogeneously colored, participants needed to maintain two attentional sets, one for each target color. In the other block (any-color), participants searched for letters that appeared in any color (except gray) within the central RSVP stream. Because all of the distractor items in the central RSVP stream were gray, participants could maintain just a single attentional set (i.e., identify any colored letter). We reason that if the enhancement effect in set size 2 is due to bottom-up perceptual priming of the target's color, then it should also be observed in any-color. On the other hand, if the enhancement effect in set size 2 stems from the fact that deeply processing a peripheral target-colored distractor leads the corresponding attentional set (e.g., identify orange letters) to enter the focus of attention, then this effect should be absent in any-color - a result that would be consistent with prior findings from a similar paradigm (Folk et al., 2008). Indeed, because all of the colors match the same attentional set in any-color, the entry of that attentional set into the focus of attention should not be associated with relatively less capture in STC than in DTC trials.

\section{Method}

Participants. Forty new individuals who had not been in Experiment 1 ( 23 female) participated in Experiment 2 for \$10. Participants (age range: 18-30 years) reported normal or corrected vision and no history of neurological injury or disease. Each consented in accordance with the University of Michigan Behavioral Sciences Institutional Review Board requirements.

Apparatus, Stimuli, and Procedure. In Experiment 2, we used the same apparatus and stimuli as in Experiment 1. In the set size 2 task block, participants performed the set size 2 task from Experiment 1 with two minor changes. First, all catch trials were replaced 
by target-alone trials because we were no longer comparing the retention of the attentional sets in set size 2 with that in set size 1 . Second, the RSVP streams were slowed slightly to $133 \mathrm{msec}$ per item in order to match the duration of distraction to that in the any-color task block, described below.

In the any-color task block, participants searched for any colored letter among gray items in the central RSVP stream, making the same judgment about target letters as they did in set size 2. Because reducing the heterogeneity of an RSVP stream results in higher target identification accuracy (Folk et al., 2002), we increased the speed of the RSVP streams in the any-color block to $33 \mathrm{msec}$ per item (with no blank space) to avoid ceiling effects. Thus, each target was presented for just $33 \mathrm{msec}$. To equate the duration of distraction in any-color and set size 2, peripheral colored distractors in the any-color block lingered for four frames, or $133 \mathrm{msec}$ (Figure 1B). To equate the temporal intervals during which distraction occurred in any-color and set size 2, peripheral colored distractors in the any-color block were presented for 0-133, 133-266, 266-400, or 400-533 msec before central targets.

There were 288 trials in the any-color block: 96 target alone, 128 DTC (4 SOAs, 32 per SOA), and 64 STC (4 SOAs, 16 per SOA). This trial distribution matched that in set size 2 , in which one third of the trials with distraction were STC. A 48-trial practice session began slowly (100 msec per frame) and accelerated gradually to $33 \mathrm{msec}$ per frame by the 24 th trial.

Analogous to Experiment 1, we counterbalanced across participants the order in which the two blocks (set size 2 and any-color) were performed, as well as the color wheel that was assigned to each block.

\section{Results}

The mean accuracy was the dependent measure in all analyses. At the outset, 6 participants ( 3 female) were eliminated because of low accuracy (i.e., below $70 \%$ in target-alone trials in either block). In the remaining 34 participants, the mean accuracy in target-alone trials did not significantly differ in the any-color $(89.9 \%)$ and set size $2(87.6 \%)$ task blocks $(p=.2)$, suggesting that participants maintained the target colors equally well in these blocks.

The results from set size 2 replicated our findings from Experiment 1. First, we observed evidence of contingent attentional capture as it is traditionally defined (i.e., worse performance in STC than in NTC trials; Figure 4A, left). Indeed, a repeated measures ANOVA with distractor type (STC, NTC) and SOA $(133,266,400,533 \mathrm{msec})$ as factors revealed a main effect of distractor type $[F(1,33)=$ $7.23, p<.011]$ because performance was worse in STC than in NTC trials, a main effect of SOA $[F(3,99)=17.1$, $p<.0001]$ because performance was worse at shorter than at longer SOAs, and an interaction between distractor type and SOA $[F(3,99)=4.46, p=.006]$ because the difference in performance between STC and NTC trials was greater at shorter than at longer SOAs. Second, a repeated measures ANOVA with distractor type (STC, DTC) and SOA $(133,266,400,533 \mathrm{msec})$ as factors revealed an enhancement effect (Figure 4A, left). More specifically, we observed a main effect of distractor type $[F(1,33)=$ $54.66, p<.0001$, indicating better performance in STC than in DTC trials; a main effect of SOA $[F(3,99)=62.9$, $p<.0001]$, indicating worse performance at shorter than at longer SOAs; and an interaction between distractor type and SOA $[F(3,99)=24.02, p<.0001]$, indicating that performance recovered from distraction more quickly in STC than in DTC trials.

We next examined performance in the any-color block to determine whether the results supported the enhancement or the priming hypothesis. As expected, the data supported the enhancement hypothesis (Figure 4A, right). In particular, the enhancement effect found in set size 2 was not observed in any-color, as indexed by the absence of a main effect of distractor type $[F(1,33)=0.22, p=.64]$ and the absence of an interaction between distractor type $(\mathrm{STC}, \mathrm{DTC})$ and SOA $[F(3,99)=1.5, p=.22]$.

Critically, we did observe evidence of attentional capture in any-color. First, there was a main effect of SOA $[F(3,99)=11.52, p<.0001]$, indicating that performance was worse at shorter than at longer SOAs. Second, the mean accuracy at the shortest SOA in trial types with target-colored distractors (i.e., mean accuracy in STC and DTC trials at the 133-msec SOA, 85.3\%) was significantly worse than the mean accuracy in target-alone trials $(89.9 \%)[t(33)=2.80, p<.008]$. Thus, as predicted, attentional capture was observed in any-color, even though the enhancement effect was not observed.

Given these results, we next investigated whether the enhancement effect was larger in set size 2 than in any-color by performing a repeated measures ANOVA with block (set size 2, any-color), distractor type (STC, DTC), and SOA $(133,266,400,533 \mathrm{msec})$ as factors. As expected, a two-way interaction between block and distractor type $[F(1,33)=18.63, p<.0001]$ indicated a significantly larger enhancement effect in set size 2 than in any-color. Moreover, a three-way interaction among block, distractor type, and SOA $[F(3,99)=11.93, p<.0001]$ revealed that the tendency for the enhancement effect to be larger at shorter than at longer SOAs was more pronounced in set size 2 than in any-color. These differences are apparent when comparing the data from set size 2 with the data from any-color in Figure 4A.

Finally, as in Experiment 1, whether the distractor and target letters were from the same or different halves of the alphabet did not affect the results. In particular, there were no main effects or interactions involving response congruency (all $p$ s > .4).

Subgroup analysis. One might wonder whether the enhancement effect was present in any-color but could not be observed statistically because the overall magnitude of attentional capture was close to floor. Indeed, attentional capture at the shortest SOA in any-color (i.e., mean accuracy in target-alone trials minus mean accuracy in STC and DTC trials) was associated with only about a $5 \%$ reduction in target identification accuracy. In contrast, an equivalent measure of attentional capture at the shortest SOA in set size 2 (i.e., capture arising from a distractor whose color matched the same attentional set as the target's color-that is, the mean accuracy in target-alone trials minus the mean accuracy in STC trials) was linked to about a $15 \%$ reduction in target identification accuracy. Thus, it is possible that we failed to observe an enhancement effect in any-color simply because target-colored distractors did not strongly capture attention. 
A

Set Size 2

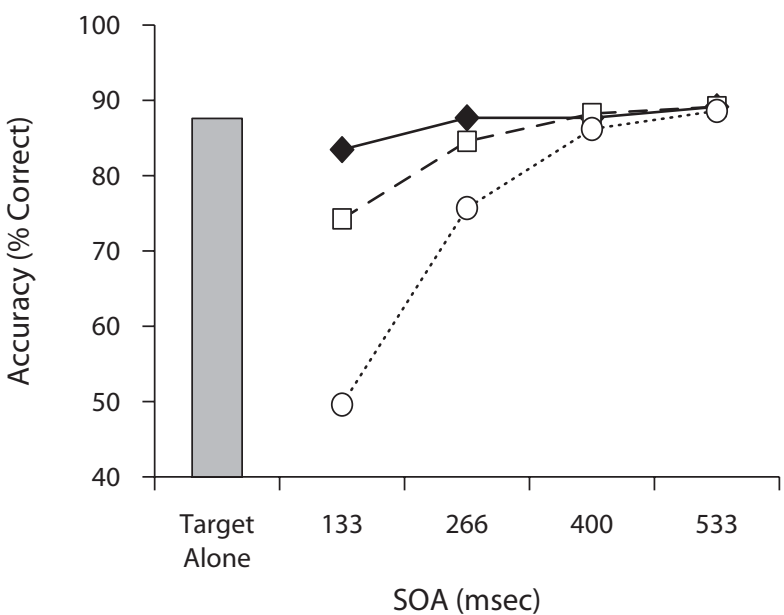

Any-Color

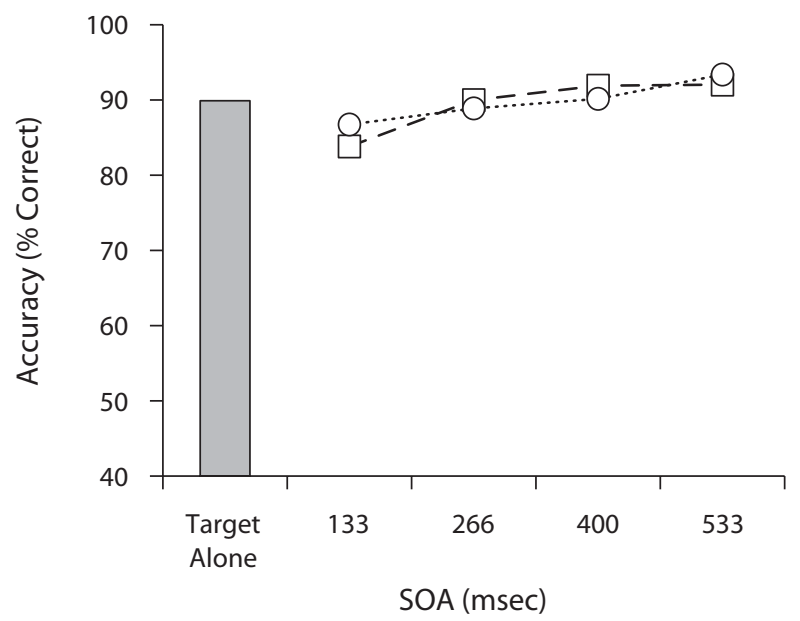

Target alone

$\sim$ NTC $\quad-\square-$ STC $\quad \cdots$ O DTC

B

Set Size 2

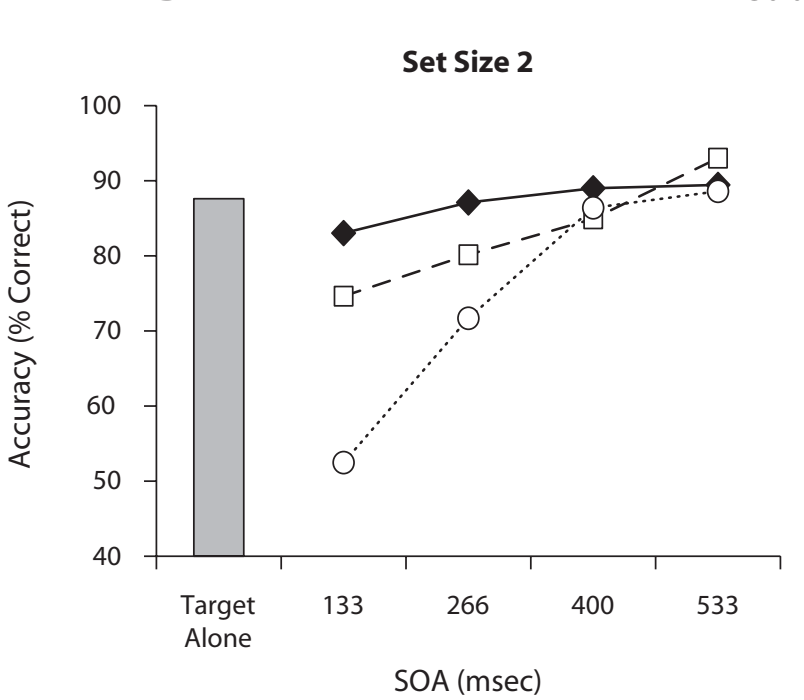

Subgroup Analysis

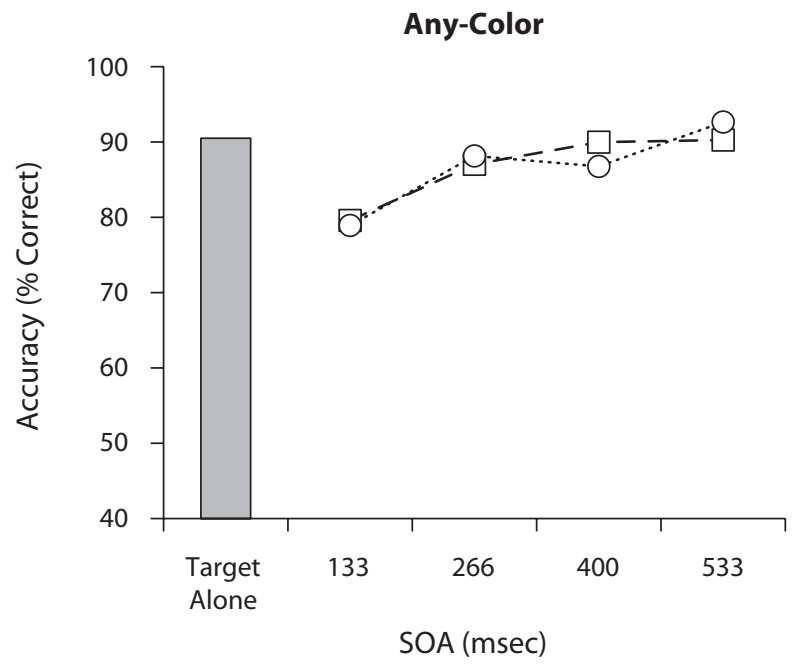

Figure 4. Data from Experiment 2. The mean target identification accuracy is plotted as a function of stimulus onset asynchrony (SOA) and distractor type in set size 2 and in any-color. (A) Consistent with the enhancement hypothesis, target identification accuracy was worse in different-target-colored (DTC) distractor trials than in same-target-colored (STC) distractor trials in set size 2, but not in any-color (B). Critically, this effect was still observed in a subgroup of participants in whom the magnitude of attentional capture in any-color (i.e., mean accuracy in target-alone trials minus accuracy in STC and DTC trials) did not differ from an equivalent measure of attentional capture in set size 2 (i.e., mean accuracy in target-alone trials minus mean accuracy in STC trials).

To evaluate this possibility, we performed additional analyses on a subgroup of our participants. The members of this subgroup were chosen as follows. First, we ranked each participant in terms of the overall size of his or her overall attentional capture effect in any-color at the shortest SOA (i.e., the mean accuracy in target-alone trials minus the mean accuracy in STC and DTC trials). Second, we selected participants for the subgroup analysis if their overall capture effect in any-color exceeded the median capture effect for the entire group. Confirming that our criteria for forming the subgroup were adequate, in this subgroup, the magnitude of attentional capture at the shortest SOA $(133 \mathrm{msec})$ was highly significant in any-color $[t(16)=5.19, p<.0001]$ (Figure 4B, right). Moreover, the magnitude of attentional capture in anycolor $(10.9 \%)$ was similar to the equivalent measure of 
attentional capture in set size 2 defined in the preceding paragraph $(12.5 \%)$. In short, we successfully created a subgroup of participants in which the attentional capture effect in any-color was relatively large.

We recognize that, due to regression of the mean, an equally large capture effect in any-color might not be observed in a follow-up experiment involving this particular subgroup of participants. However, the goal of the subgroup analysis was simply to assess whether an enhancement effect in any-color could be observed when attentional capture effects were relatively large and, consequently, any potential ceiling effects on the size of the enhancement effect were removed. Thus, the subgroup analysis was appropriate for achieving this objective.

It is also important for us to note that our selection criteria for membership in the subgroup and our subsequent analyses of the enhancement effect were orthogonal. Selection into the subgroup was based on having a large overall capture effect in the any-color condition, as defined by the difference between target-alone performance and the average of STC and DTC performance at the shortest SOA. In contrast, the enhancement effect was defined by the difference between the STC and DTC trial types. Thus, our method of choosing individuals for the subgroup analysis did not bias the results of our subsequent analyses of the enhancement effect in these participants, either in anycolor or in set size 2 .

As expected, the subgroup analysis replicated the main findings from the overall analysis. First, a repeated measures ANOVA with distractor type (STC, DTC) and SOA $(133,266,400,533 \mathrm{msec})$ as factors and the mean accuracy in any-color as the dependent measure revealed no main effect of distractor type $[F(1,16)=0.009, p=.925]$ and a main effect of SOA $[F(3,48)=15.43, p<.0001]$, because performance was worse at shorter than at longer SOAs. Second, a repeated measures ANOVA with distractor type (STC, DTC) and SOA (133, 266, 400, $533 \mathrm{msec})$ as factors and mean accuracy in set size 2 as the dependent measure revealed significant main effects of distractor type $[F(1,16)=13.24, p<.002]$ and SOA $[F(3,48)=$ $26.94, p<.0001]$, as well as an interaction between distractor type and SOA $[F(3,48)=5.50, p<.002]$. In short, as in the overall analysis, and consistent with the enhancement hypothesis, in set size 2, performance was worse in DTC than in STC trials, and this difference was greater at shorter than at longer SOAs; in contrast, in any-color, no such effects were observed.

Critically, the subgroup analysis also replicated our finding of a significantly smaller enhancement effect in any-color than in set size 2. A repeated measures ANOVA with block (any-color, set size 2), distractor type (STC, DTC), and SOA (133, 266, 400, $533 \mathrm{msec})$ as factors revealed a two-way interaction between block and trial type $[F(1,16)=8.13, p<.012]$ and a three-way interaction among block, trial type, and SOA $[F(3,48)=5.03, p<$ .004] (Figure 4B). Put simply, as in the overall analysis, the difference in performance between STC and DTC trials was significantly larger in set size 2 than in any-color, and this effect was more pronounced at shorter than at longer SOAs. Thus, in line with our hypothesis, the enhancement effect was significantly larger in set size 2 than in any-color, even in participants who exhibited relatively large capture effects in any-color.

Finally, as in the overall analysis, there were no main effects or interactions involving response congruency (all ps >.4).

\section{Discussion}

Our findings from Experiment 2 further support the enhancement hypothesis while weighing against the possibility that the enhancement effect in set size 2 stems from bottom-up perceptual priming of the distractor's color. First, we replicated the enhancement effect observed in Experiment 1. That is, in set size 2, performance was better in STC than in DTC trials. Second, the enhancement effect was absent in any-color, in which all of the colors matched the same attentional set. Third, and critically, the enhancement effect was significantly smaller in any-color than in set size 2, even when the overall magnitude of attentional capture was matched in these task blocks within a subgroup of the participants. Taken together, these results indicate that the enhancement effect found in set size 2 was not due solely to bottom-up perceptual priming of the distractor's color. Thus, our findings in Experiment 2 further support the enhancement hypothesis.

\section{EXPERIMENT 3}

In Experiment 3, we investigated another alternative account of the enhancement effect in set size 2. According to this alternative account, better performance in STC than in DTC trials occurs because there is confusion about the location of the target in STC trials, but about both the location and the color of the target in DTC trials. In other words, the target-colored distractor activates a representation of an incorrect location in STC trials, but it activates a representation of an incorrect location and a representation of an incorrect color in DTC trials. Given that there are fewer sources of confusion, or interference, in STC than in DTC trials, this interference-based account predicts better performance in STC than in DTC trials and, therefore, appears to explain our findings as well as the enhancement hypothesis.

In Experiment 3, we distinguished between these competing accounts by varying whether the target-colored distractor was presented before or after the critical target. Although both accounts predict better performance in STC than in DTC trials when the critical distractor appears before the target, they make different predictions about performance in STC and DTC trials when the critical distractor appears after the target. The interferencebased account still predicts better performance in STC than in DTC trials because interference is always lower in STC than in DTC trials. In contrast, the enhancement hypothesis predicts better performance in DTC than in STC trials. According to this hypothesis, detecting a potential target leads the attentional set corresponding to that item's color to enter into the focus of attention. Thus, 
a target-colored distractor that appears immediately after the target should be detected more readily, and hence interfere with performance to a greater degree, when its color matches the same attentional set as the target than when its color matches a different attentional set. In sum, the goal of Experiment 3 was to contrast two accounts of the enhancement effect by comparing performance in STC and DTC trials when a target-colored distractor was presented after a target.

\section{Method}

Participants. Thirty-six new individuals who had not participated in either Experiment 1 or Experiment 2 (20 female) participated in Experiment 3 for $\$ 10$ or course credit. Participants (age range: 18-25 years) reported normal or corrected vision and no history of neurological injury or disease. Each gave informed written consent in accordance with the requirements of the University of Michigan Behavioral Sciences Institutional Review Board.

Apparatus, Stimuli, and Procedure. We used the same apparatus and stimuli as in Experiments 1 and 2. Colors for the letter stimuli were drawn from the light color wheel only; the dark color wheel was not used in this experiment.

The procedure was the same as that for the set size 2 condition of Experiment 1, with three exceptions. First, we replaced the 350and 466-msec SOA trial types with -116- and -233-msec SOA trial types. Thus, in half of the trials, the distractor appeared before the target (the 116- and 233-msec SOA trial types), whereas in the other half, the distractor appeared after the target (the -116- and -233 -msec SOA trial types). Second, we doubled the number of trials in each trial type to increase statistical power. Third, as in Experiment 2, we did not include catch trials, so we used target-alone performance as a criterion for including participants. In total, there were 576 trials: 192 target alone, 128 NTC ( 32 per SOA), 128 STC (32 per SOA), and 128 DTC (32 per SOA).

\section{Results}

The mean percent correct was the dependent measure in all of our analyses. At the outset, we excluded 8 participants with poor performance (target-alone accuracy below 50\%). We set a lower threshold for exclusion in Experiment 3 than in Experiment 2 for two reasons. First, we reasoned that participants who were less skilled at this task might experience greater capture from a targetcolored distractor (either STC or DTC) that appeared after a target - an effect that needed to be present for us to test our hypotheses in Experiment 3. Second, as a group, the participants in Experiment 3 performed more poorly than those in Experiments 1 and 2.

First, we conducted two separate repeated measures ANOVAs in order to examine whether we replicated the capture and enhancement effects found in Experiments 1 and 2. The first ANOVA had two factors: distractor type (NTC, STC) and SOA (116, $233 \mathrm{msec})$. This ANOVA showed that we replicated the basic contingent attentional capture effect when the target-colored distractor appeared before the target. More specifically, it revealed main effects of distractor type $[F(1,27)=4.44, p<.045]$ and SOA $[F(1,27)=7.87, p<.009]$, as well as a distractor type $\times$ SOA interaction $[F(1,27)=4.71, p<.039]$. As in Experiments 1 and 2, performance was worse in STC than in NTC trials, and this difference was larger at the 116-msec SOA than at the 233-msec SOA (Figure 5, right).
The second ANOVA also had two factors: distractor type (STC, DTC) and SOA (116, $233 \mathrm{msec})$. This ANOVA showed that we replicated the enhancement effect when the target-colored distractor appeared before the target. Specifically, it revealed main effects of distractor type $[F(1,27)=33.00, p<.0001]$ and SOA $[F(1,27)=23.65$, $p<.0001]$, as well as a distractor type $\times$ SOA interaction $[F(1,27)=15.24, p<.001]$. Consistent with Experiments 1 and 2, performance was better in STC than in DTC trials, and this difference was greater at the 116-msec SOA than at the 233-msec SOA (Figure 5, right).

Next, we performed the critical analysis needed to distinguish between the enhancement and interference hypotheses. As uniquely predicted by the enhancement hypothesis, an ANOVA with the factors distractor type (STC, DTC) and SOA $(-116,-233 \mathrm{msec})$ revealed a significant reversed enhancement effect; that is, performance was worse in STC than in DTC trials $[F(1,27)=7.06, p<$ .013] (Figure 5, left).

It is important for us to note that the aforementioned reversed enhancement effect was caused by a selective performance decrement in STC trials. First, an ANOVA with the factors distractor type (NTC, STC) and SOA $(-116,-233 \mathrm{msec})$ revealed a significant main effect of distractor type $[F(1,27)=7.12, p<.013]$ because performance was significantly worse in STC than in NTC trials. Second, an ANOVA with the factors distractor type (NTC, DTC) and SOA $(-116,-233 \mathrm{msec})$ did not reveal a main effect of distractor type $[F(1,27)=0.02, p=.874]$ because performance did not differ in DTC and NTC trials. Together, these results suggest that contingent attentional capture involves both an increase of attention to a potential target's attentional set and a decrease of attention to other attentional sets.

Finally, as in Experiments 1 and 2, there were no main effects or interactions involving response congruency (all $p \mathrm{~s}>.5)$.

\section{Discussion}

The results of Experiment 3 further support the enhancement hypothesis while ruling out an interferencebased account of the enhancement effect. As in Experiments 1 and 2, performance was better in STC than in DTC trials when the target-colored distractor appeared before the target, indicating an enhancement effect. Critically, however, the enhancement effect was reversed when the target-colored distractor appeared after the target. This result is inconsistent with the interference hypothesis, which predicts less interference (and thus better performance) in STC than in DTC trials, regardless of whether the target-colored distractor is presented before or after the target. In contrast, this result is consistent with the enhancement hypothesis. In particular, if the target appears before the target-colored distractor, then the entry of its attentional set into the focus of attention should facilitate the detection of a subsequent distractor if its color matches the same (vs. a different) attentional set. In sum, the results of Experiment 3 provide further support for the enhancement hypothesis. 


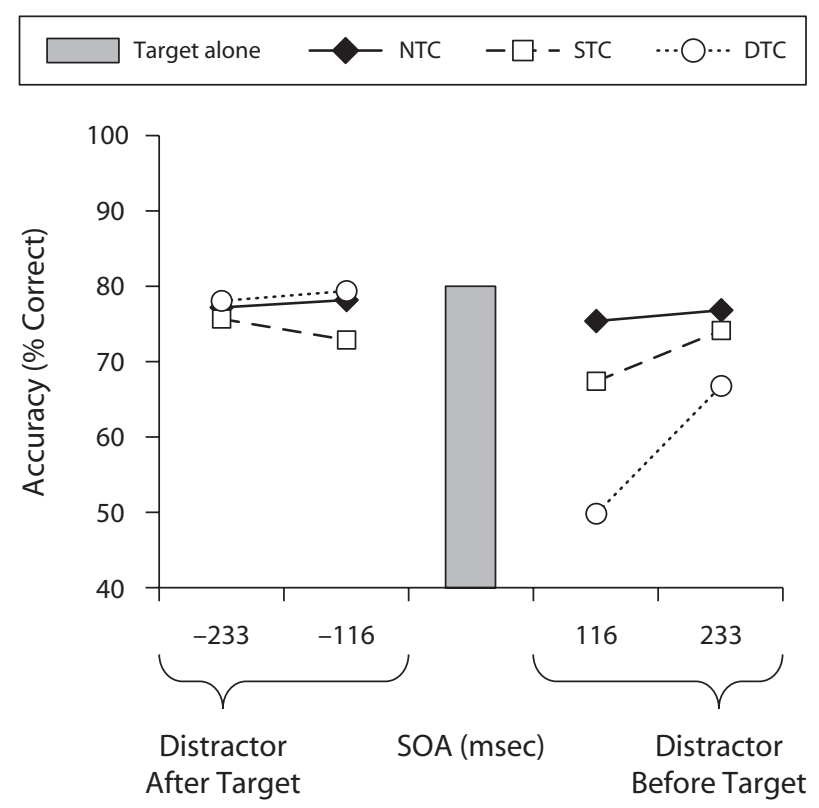

Figure 5. Data from Experiment 3. The mean target identification accuracy is plotted as a function of stimulus onset asynchrony (SOA) and distractor type. When the distractor appeared prior to the target (positive SOAs; right side of the figure), performance was better in non-target-colored (NTC) than in same-targetcolored (STC) trials, indicating contingent attentional capture. Performance was also better in STC than in different-targetcolored (DTC) trials, indicating an enhancement effect. In each of these trial types, performance was better at the longer (233-msec) than at the shorter (116-msec) SOA, likely because participants had more time to recover from distraction. When the distractor appeared after the target (negative SOAs; left side of the figure), performance was worse in STC than in either NTC or DTC trials, as predicted by the enhancement hypothesis. Performance was better at the longer $(-233-\mathrm{msec})$ than at the shorter $(-116-\mathrm{msec})$ SOA, probably because target identification was more likely to be completed before the critical distractor appeared at the longer than at the shorter SOA.

\section{GENERAL DISCUSSION}

Although selective attention usually helps to prevent irrelevant stimuli from gaining access to limited-capacity information processing systems, it occasionally has the opposite effect (Folk et al., 1992). For example, distractors that possess a target-defining color attract attention and are deeply processed in a limited-capacity manner (Folk et al., 2002; Serences et al., 2005). In the present study, we investigated whether such deep processing of a targetcolored distractor causes the corresponding attentional set to enter a limited-capacity focus of attention within working memory (Jonides et al., 2008; Oberauer, 2002), leading to enhanced identification of a subsequent target whose features match the same attentional set. Findings from three experiments supported this enhancement hypothesis. Moreover, they weighed against two alternative accounts of our results.

In Experiment 1, we both replicated previous findings of contingent attentional capture and observed initial support for the enhancement hypothesis. First, consistent with prior studies of contingent attentional capture, in both set sizes, target identification accuracy was lower when a target was preceded by an STC distractor than when it was preceded by an NTC distractor, and this effect was larger at shorter than at longer SOAs (Folk et al., 2002, 2008). Such effects were similar in magnitude in set size 1 and set size 2, consistent with the data from catch trials suggesting that participants were able to maintain one and two attentional sets equally well. Second, in line with the enhancement hypothesis, target identification accuracy was higher when a target was preceded by an STC distractor than when it was preceded by a DTC distractor, and this effect was larger at shorter than at longer SOAs. This enhancement effect suggests that deeply processing a target-colored distractor causes the corresponding attentional set to enter a focus of attention within working memory that can hold just a single item (Jonides et al., 2008; Oberauer, 2002, 2003).

In Experiment 2, we ruled out bottom-up perceptual priming as an alternative explanation for the enhancement effect. Performance was measured in two task blocks. In one task block (set size 2), participants identified letters in the central RSVP stream that possessed either of two target colors while ignoring letters that possessed other colors. The presence of irrelevant colors in the central RSVP stream made it necessary for participants to maintain two attentional sets, one for each target color. In the other task block (any-color), participants identified any colored letter in the central RSVP stream. Thus, they could maintain just a single attentional set, and all colors matched the same set. In line with the enhancement hypothesis, the enhancement effect was highly robust when different colors matched distinct attentional sets in set size 2, but was completely absent when different colors matched the same attentional set in any-color. This finding weighs against the possibility that the enhancement effect stems solely from bottom-up perceptual priming of the distractor's color.

However, it is important that one consider whether differences in the number of attentional sets that participants maintained in set size 2 (two sets) and in any-color (one set) in Experiment 2 might have caused the enhancement effect to be larger in set size 2 than in any-color. For example, contingent attentional capture effects might simply scale with the number of attentional sets that participants maintain. Arguing against this possibility, in Experiment 1 , we did not observe a significant difference in the magnitude of contingent attentional capture in set size 1 and set size 2. In fact, contrasting performance in STC and NTC trials revealed numerically less contingent attentional capture in set size 2 than in set size 1. For this reason, differences in the number of attentional sets that participants maintained do not appear to account for our finding that the enhancement effect was larger in set size 2 than in any-color.

In Experiment 3, we ruled out an interference-based account of the enhancement effect in set size 2. According to this account, performance was better in STC than in DTC trials because there was confusion, or interference, with 
regard to the location of the target in STC trials, but with regard to both the location and the color of the target in DTC trials. To pit this interference-based account against the enhancement hypothesis, we varied whether the targetcolored distractor was presented before or after the target. As in Experiments 1 and 2, when the target-colored distractor was presented before the target in set size 2, performance was better in STC than in DTC trials - a result that could be explained by either hypothesis. However, when the target-colored distractor was presented after the target, performance was worse in STC than in DTC trials - a result that could be explained only by the enhancement hypothesis. Thus, our findings in Experiment 3 not only rule out an interference-based account of the enhancement effect, but also provide converging support for the enhancement hypothesis.

Although the results of Experiments 2 and 3 weigh against various alternative accounts of our findings, one might question how strongly they actually support the enhancement hypothesis. Indeed, evidence for the enhancement hypothesis was observed as a reduction of contingent attentional capture costs, rather than as an improvement in performance relative to conditions that did not contain target-colored distractors. However, it is standard practice in the literature to show that an effect is present by demonstrating that it modulates the size of a performance decrement. For example, in studies of task switching, an effect of task set reconfiguration has been revealed by showing that switch costs can be reduced, but not fully eliminated, by giving participants more time to prepare for an upcoming task switch (Meiran, Chorev, \& Sapir, 2000). Analogously, in the present study of contingent attentional capture, an effect of enhancing a potential target's attentional set has been demonstrated by showing that the magnitude of capture varies with whether a distractor's color and a target's color match the same or different attentional sets. Thus, our findings support the enhancement hypothesis, even though effects of enhancement manifested themselves as a reduction of contingent attentional capture costs rather than as an improvement in performance relative to conditions without target-colored distractors.

Given the robust enhancement effects that we have observed, it would appear that most prior studies have failed to appreciate the full range of contingent attentional capture effects. In almost all contingent attentional capture studies, participants maintain just a single attentional set (Folk et al., 2002; Folk et al., 1992; Serences et al., 2005; Shih \& Reeves, 2007), which means that contingent capture effects arising from a target-resembling distractor are estimated when the attentional set that defines the subsequent target is already within the focus of attention. Under these conditions, such capture effects index not only a reduction of target identification accuracy that is caused by deeply processing a target-resembling distractor, but also an enhancement of target identification accuracy that is caused by the presence of the target-defining attentional set within the focus of attention. Only by requiring participants to maintain two distinct attentional sets in set size 2 were we able to measure contingent attentional capture effects in the absence of such enhancement. Notably, contingent attentional capture effects at short SOAs were two to three times larger when the target-defining attentional set was outside the focus of attention (i.e., in DTC trials) than when it was within the focus of attention (i.e., in STC trials) (see the data from set size 2 in Figures 3, 4, and 5). Thus, contingent attentional capture effects can sometimes be much more pronounced than previous studies have indicated.

The enhancement effect is consistent with other data indicating that transferring a task or attentional representation into the focus of attention enhances subsequent behaviors involving that representation. As we discussed in the introduction, this view is supported by prior studies of working memory (Berti, 2008; Garavan, 1998; McElree, 2001), task switching (Hsieh \& Allport, 1994; Monsell, 2003), and the attentional blink (Juola et al., 2004; Vachon et al., 2007). For example, in the attentional blink paradigm, the second of two targets is identified more accurately when it matches the same conceptual category (e.g., digits) as the first target than when it matches a different category (e.g., letters) (Juola et al., 2004). Our findings suggest that identifying the first target causes the corresponding attentional set (e.g., identify digits) to be transferred into the focus of attention, leading to a smaller attentional blink when the second target is identified using the same (vs. a different) attentional set. Moreover, they indicate that even an irrelevant, target-resembling distractor can cause an attentional set to be transferred into the limited-capacity focus of attention, from which it can enhance subsequent behaviors relying on the same set.

As mentioned previously, the enhancement effect may appear to conflict with prior data indicating that attentional sets can be primed across trials. For example, although intertrial priming of top-down attentional sets is not always observed (Ansorge \& Horstmann, 2007; Ansorge et al., 2005), identifying a distractor that possesses a target-defining color sometimes decreases an attentional bias toward that color in the next trial (Lleras et al., 2009). On the basis of such findings, one might have predicted worse performance in the present experiments when a target possessed the same color as a preceding distractor in STC trials than when it possessed a different color in DTC trials, because of a reduction of attention to the distractor's color. However, given that the detection of a target-colored distractor initially leads that item to be deeply processed as though it were a target (Folk et al., 2002), an attentional bias toward that item's color may increase for a brief time (Dux \& Marois, 2009), even if it is subsequently reduced. Converging evidence for such an increase comes from the finding that presenting a distractor just before presenting the second of two targets in an RSVP stream reduces the magnitude of the attentional blink most strongly when the distractor possesses the upcoming target's color (Nieuwenstein, 2006; Nieuwenstein, Chun, van der Lubbe, \& Hooge, 2005). Thus, any perceived discrepancy between our findings and previous work revealing intertrial priming of topdown attentional sets (Folk \& Remington, 2008; Lleras 
et al., 2009) likely stems from the relatively short interval over which our within-trial effects were measured.

Although we have argued that the enhancement effect stems from a limited-capacity focus of attention, it is worthwhile to consider whether it might alternatively arise from a brief lapse of attention. This possibility is suggested by the temporary loss of control (TLC) model, which was first developed to explain various phenomena in the attentional blink paradigm (Di Lollo, Kawahara, Ghorashi, \& Enns, 2005). In this model, a limited-capacity central processor biases attentional filters to detect stimuli that possess one or more target-defining features (e.g., task-relevant colors). When such a stimulus is detected, it is directed to the central processor for purposes of stimulus identification, during which time the central processor does not have sufficient resources to continue biasing the attentional filters. Thus, until the potential target is identified, these filters are exogenously reconfigured by each subsequent distractor item that appears, leading to exogenous (rather than endogenous) changes in attentional set.

Of importance, the TLC model cannot explain our finding that performance was better in STC than in DTC trials when the target-colored peripheral distractor and the subsequent target were separated by one or more colored distractors in the central RSVP stream. The TLC model posits that by the time a target appears in such trials, attentional filters should already be reconfigured to match the color of the central RSVP stream distractor that immediately preceded the target (for similar reasoning, see Nieuwenstein, 2006). Thus, identifying the target should require a time-consuming change of attentional set in both STC and DTC trials, leading to equivalent performance in these trial types. Given that performance was better in STC than in DTC trials, even when the target-colored distractor and the subsequent distractor were separated by one or more colored distractors, the TLC model does not appear able to account for the enhancement effect.

Although we have emphasized enhancement when accounting for the present data, inhibitory mechanisms may also play a role. For example, our finding that target identification accuracy was lower when the target's color matched a different (vs. the same) attentional set than the preceding distractor's color may stem, in part, from a distractor-triggered inhibition of competing attentional sets. In other words, when searching for orange and green target letters, detecting an orange distractor may result not only in transferring the "orange" attentional set into the focus of attention, but also in inhibiting the "green" attentional set. Consistent with this possibility, task switching appears to involve not only loading a new task set into the focus of attention, but also inhibiting the previous task set (Mayr \& Keele, 2000). Given that the present experiments were not designed to distinguish between enhancement and inhibition, however, future work will be necessary to determine the relative contributions of these processes to the enhancement effect.

Our account of the enhancement effect relies on the assumption that only a single representation can occupy the focus of attention (Oberauer, 2002, 2003). However, it is well established that multiple items can be maintained within the focus of attention when they are chunked, or bound, into a single representation (Jonides et al., 2008; Oberauer \& Bialkova, 2009). Consistent with this view, the behavioral cost associated with switching attention between items in working memory is eliminated when those items are bound into a single object representation. For example, when updating a location and a count in working memory, participants experience no cost of switching between these representations when they are bound by imagining a moving number (Bao, Li, \& Zhang, 2007). This result suggests that the enhancement effect might be absent if distinct attentional sets could be bound into a single representation. Although additional studies are necessary to test this hypothesis, such a result would provide complementary evidence that contingent attentional capture effects stem from a limited-capacity focus of attention within working memory.

More broadly, the present findings may have important implications for real-world activities that involve maintaining multiple attentional sets (see, e.g., Most \& Astur, 2007). For example, while driving to brunch on a winding highway, we may simultaneously be searching for a yellow warning sign indicating an upcoming twist in the road and a restaurant billboard that has either the same color as (e.g., yellow, Waffle House) or a different color than (e.g., blue, International House of Pancakes) the warning sign. The present findings suggest that drivers would be much more likely to miss such a warning sign when it appears in a different (vs. the same) color as an immediately preceding, target-colored billboard. Given that nearly $80 \%$ of car crashes are immediately preceded by a moment of driver inattention (Ranney, 2008), applied research should be aimed at identifying and minimizing contingent attentional capture effects in real-world settings.

In sum, our findings indicate that involuntarily directing attention to a distractor that possesses a targetdefining color (e.g., orange) leads the corresponding attentional set (e.g., identify orange letters) to enter a limited-capacity focus of attention within working memory, thereby enhancing the identification of a subsequent target whose color matches the same (vs. a different) attentional set. Specifically, contingent attentional capture effects were only one half to one third as large when detecting a target relied on the same (vs. a different) attentional set as detecting a preceding target-colored distractor. Of importance, neither bottom-up perceptual priming nor feature-based interference could account for this modulation of contingent attentional capture effects. Future work investigating the possible influences of inhibitory mechanisms and chunking on the enhancement effect may reveal additional information about how capacity limitations in working memory contribute to contingent attentional capture effects, both in the laboratory and in real-world situations.

\section{AUTHOR NOTE}

This research was supported by a National Science Foundation graduate fellowship, a Rackham Graduate Research Award, and a Pillsbury award 
to K.S.M., as well as by start-up funds from the University of Michigan's Department of Psychology and an NIH grant (1R03DA021345-01) awarded to D.H.W. The authors thank Amanda Lai, Marshall O'Moore, Patricia Chen, Jillian Steinberg, and Anna Grummon for assistance with data collection. We also thank Ulrich Ansorge, Artem Belopolsky, Andy Leber, Bradley Gibson, Joshua Carp, and Kristin Flegal for their thoughtful comments on earlier drafts of the manuscript. Finally, we wish to thank Randy Buckner and Alejandro Lleras for useful discussions that led to Experiments 2 and 3. Correspondence concerning this article should be addressed to K. S. Moore, Department of Psychology, University of Michigan, 1012 East Hall, 530 Church Street, Ann Arbor, MI 48109 (e-mail: mooreks@umich.edu).

\section{REFERENCES}

Adamo, M., Pun, C., Pratt, J., \& Ferber, S. (2008). Your divided attention, please! The maintenance of multiple attentional control sets over distinct regions in space. Cognition, 107, 295-303.

Anderson, B. A., \& FolK, C. L. (2010). Variations in the magnitude of attentional capture: Testing a two-process model. Attention, Perception, \& Psychophysics, 72, 342-352.

Ansorge, U., \& Heumann, M. (2003). Top-down contingencies in peripheral cuing: The roles of color and location. Journal of Experimental Psychology: Human Perception \& Performance, 29, 937-948.

Ansorge, U., \& Horstmann, G. (2007). Preemptive control of attentional capture by colour: Evidence from trial-by-trial analyses and orderings of onsets of capture effects in reaction time distributions. Quarterly Journal of Experimental Psychology, 60, 952-975.

Ansorge, U., Horstmann, G., \& Carbone, E. (2005). Top-down contingent capture by colour: Evidence from RT distribution analyses in a manual choice reaction task. Acta Psychologica, 120, 243-266.

BACON, W. F., \& EgETH, H. E. (1994). Overriding stimulus-driven attentional capture. Perception \& Psychophysics, 55, 485-496.

BAO, M., LI, Z.-H., \& ZHANG, D.-R. (2007). Binding facilitates attention switching within working memory. Journal of Experimental Psychology: Learning, Memory, \& Cognition, 33, 959-969.

Belopolsky, A. V., Kramer, A. F., \& Godijn, R. (2008). Transfer of information into working memory during attentional capture. Visual Cognition, 16, 409-418.

Belopolsky, A. V., SchreiJ, D., \& Theeuwes, J. (2010). What is topdown about contingent capture? Attention, Perception, \& Psychophysics, 72, 326-341.

BERTI, S. (2008). Object switching within working memory is reflected in the human event-related brain potential. Neuroscience Letters, $\mathbf{4 3 4}$, 200-205.

Corbetta, M., \& Shulman, G. L. (2002). Control of goal-directed and stimulus-driven attention in the brain. Nature Reviews Neuroscience, 3, 201-215

Cowan, N. (2001). The magical number 4 in short-term memory: A reconsideration of mental storage capacity. Behavioral \& Brain Sciences, 24, 87-185.

Desimone, R., \& Duncan, J. (1995). Neural mechanisms of selective visual attention. Annual Review of Neuroscience, 18, 193-222.

Di Lollo, V., KaWAhara, J., Ghorashi, S. M. S., \& EnNS, J. T. (2005). The attentional blink: Resource depletion or temporary loss of control? Psychological Research, 69, 191-200.

Dux, P. E., \& MARoIs, R. (2009). The attentional blink: A review of data and theory. Attention, Perception, \& Psychophysics, 71, 1683-1700.

FARELL, B. (1985). Same-different judgments: A review of current controversies in perceptual comparisons. Psychological Bulletin, 98, 419-456.

Folk, C. L., Leber, A. B., \& Egeth, H. E. (2002). Made you blink! Contingent attentional capture produces a spatial blink. Perception \& Psychophysics, 64, 741-753.

Folk, C. L., Leber, A. B., \& Egeth, H. E. (2008). Top-down control settings and the attentional blink: Evidence for nonspatial contingent capture. Visual Cognition, 16, 616-642.

Folk, C. L., \& Remington, R. W. (2008). Bottom-up priming of topdown attentional control settings. Visual Cognition, 16, 215-231.

Folk, C. L., Remington, R. W., \& Johnston, J. C. (1992). Involuntary covert orienting is contingent on attentional control settings. Journal of Experimental Psychology: Human Perception \& Performance, 18, 1030-1044
Garavan, H. (1998). Serial attention within working memory. Memory \& Cognition, 26, 263-276.

Gibson, B. S., \& Kelsey, E. M. (1998). Stimulus-driven attentional capture is contingent on attentional set for displaywide visual features. Journal of Experimental Psychology: Human Perception \& Performance, 24, 699-706.

Henson, R. N. A. (2003). Neuroimaging studies of priming. Progress in Neurobiology, 70, 53-81.

HoutKamp, R., \& RoELFSEMa, P. R. (2006). The effect of items in working memory on the deployment of attention and eyes during visual search. Journal of Experimental Psychology: Human Perception \& Performance, 32, 423-442.

Hsieh, S., \& Allport, A. (1994). Shifting attention in a rapid visual search paradigm. Perceptual \& Motor Skills, 79, 315-335.

Jonides, J., Lewis, R. L., Nee, D. E., Lustig, C. A., Berman, M. G., \& Moore, K. S. (2008). The mind and brain of short-term memory. Annual Review of Psychology, 59, 193-224.

Juola, J. F., Botella, J., \& Palacios, A. (2004). Task- and locationswitching effects on visual attention. Perception \& Psychophysics, 66, 1303-1317.

Leber, A. B., \& Egeth, H. E. (2006). Attention on autopilot: Past experience and attentional set. Visual Cognition, 14, 565-583.

Leblanc, E., Prime, D. J., \& Jolicceur, P. (2008). Tracking the location of visuospatial attention in a contingent capture paradigm. Journal of Cognitive Neuroscience, 20, 657-671.

Lleras, A., Kawahara, J., \& Levinthal, B. R. (2009). Past rejections lead to future misses: Selection-related inhibition produces blinklike misses of future (easily detectable) events. Journal of Vision, 9(3, Art. 26), 1-12.

Mayr, U., \& Keele, S. W. (2000). Changing internal constraints on action: The role of backward inhibition. Journal of Experimental Psychology: General, 129, 4-26.

MCELREE, B. (2001). Working memory and focal attention. Journal of Experimental Psychology: Learning, Memory, \& Cognition, 27, 817-835.

Meiran, N., Chorev, Z., \& Sapir, A. (2000). Component processes in task switching. Cognitive Psychology, 41, 211-253.

Monsell, S. (2003). Task switching. Trends in Cognitive Sciences, 7 , 134-140.

Most, S. B., \& Astur, R. S. (2007). Feature-based attentional set as a cause of traffic accidents. Visual Cognition, 15, 125-132.

Nieuwenstein, M. R. (2006). Top-down controlled, delayed selection in the attentional blink. Journal of Experimental Psychology: Human Perception \& Performance, 32, 973-985.

Nieuwenstein, M. R., Chun, M. M., van der Lubbe, R. H. J., \& Hooge, T. C. (2005). Delayed attentional engagement in the attentional blink. Journal of Experimental Psychology: Human Perception \& Performance, 31, 1463-1475.

OBERAUER, K. (2002). Access to information in working memory: Exploring the focus of attention. Journal of Experimental Psychology: Learning, Memory, \& Cognition, 28, 411-421.

Oberauer, K. (2003). Selective attention to elements in working memory. Experimental Psychology, 50, 257-269.

OBERAUER, K., \& BialKova, S. (2009). Accessing information in working memory: Can the focus of attention grasp two elements at the same time? Journal of Experimental Psychology: General, 138, 64-87.

OLIVERS, C. N. L. (2008). Interactions between visual working memory and visual attention. Frontiers in Bioscience, 13, 1182-1191.

Olivers, C. N. L. (2009). What drives memory-driven attentional capture? The effects of memory type, display type, and search type. Journal of Experimental Psychology: Human Perception \& Performance, 35, 1275-1291.

Olivers, C. N. L., \& Humphreys, G. W. (2003). Attentional guidance by salient feature singletons depends on intertrial contingencies. Journal of Experimental Psychology: Human Perception \& Performance, 29, 650-657.

Olivers, C. N. L., \& Meeter, M. (2008). A boost and bounce theory of temporal attention. Psychological Review, 115, 836-863.

Posner, M. I., \& Mitchell, R. F. (1967). Chronometric analysis of classification. Psychological Review, 74, 392-409.

Posner, M. I., \& Rothbart, M. K. (2007). Research on attention networks as a model for the integration of psychological science. Annual Review of Psychology, 58, 1-23.

RanNEY, T. A. (2008). Driver distraction: A review of the current state- 
of-knowledge. Washington, DC: National Highway Traffic Safety Administration.

Serences, J. T., Shomstein, S., Leber, A. B., Golay, X., Egeth, H. E., \& YANTIS, S. (2005). Coordination of voluntary and stimulus-driven attentional control in human cortex. Psychological Science, 16, 114122.

ShiH, S.-I., \& Reeves, A. (2007). Attentional capture in rapid serial visual presentation. Spatial Vision, 20, 301-315.

Soto, D., Heinke, D., Humphreys, G. W., \& Blanco, M. J. (2005). Early, involuntary top-down guidance of attention from working memory. Journal of Experimental Psychology: Human Perception \& Performance, 31, 248-261.

Thompson, C., Underwood, G., \& Crundall, D. (2007). Previous attentional set can induce an attentional blink with task-irrelevant initial targets. Quarterly Journal of Experimental Psychology, 60, 1603-1609.
Vachon, F., Tremblay, S., \& Jones, D. M. (2007). Task-set reconfiguration suspends perceptual priming: Evidence from semantic priming during the attentional blink. Journal of Experimental Psychology: Human Perception \& Performance, 33, 330-347.

Visser, T. A. W., Bischof, W. F., \& Di Lollo, V. (2004). Rapid serial visual distraction: Task-irrelevant items can produce an attentional blink. Perception \& Psychophysics, 66, 1418-1432.

Woodman, G. F., \& LUCK, S. J. (2007). Do the contents of visual working memory automatically influence attentional selection during visual search? Journal of Experimental Psychology: Human Perception \& Performance, 33, 363-377.

Yantis, S., \& Egeth, H. E. (1997). Visual attention: Control, representation, and time course. Annual Review of Psychology, 48, 269-297.

(Manuscript received August 14, 2009; revision accepted for publication April 12, 2010.) 